\title{
Caracterización, innovación y competitividad de la producción de elotes en el Estado de Jalisco, México
}

\section{Characterization, innovation and competitiveness of the production of corn of the cob in the state of Jalisco, México}

DOI: https://doi.org/10.17981/econcuc.41.2.2020.Org.3

Artículo de investigación.

Fecha de recepción: 10/12/2019

Fecha de devolución: 15/03/2020

Fecha de aceptación: 31/03/2020

Fecha de publicación: 07/04/2020

\section{Anastacio Espejel García iD \\ Universidad Autónoma Chapingo. \\ Texcoco (México) \\ anastacio.espejel@gmail.com}

\section{Carla Zulema Jauregui García \\ Universidad Autónoma Chapingo. \\ Texcoco (México) \\ al19132146@chapingo.mx}

\section{Arturo Hernández Montes (iD \\ Universidad Autónoma Chapingo. \\ Texcoco (México) sensorial@prodigy.net.mx}

Para citar este artículo:

Espejel, A., Jauregui, C. \& Hernández, A. (2020).

Caracterización, innovación y competitividad de la producción de elotes en el Estado de Jalisco, México. Económicas CUC, 41(2), 49-64. DOI: https://doi.org/10.17981/econcuc.41.2.2020.Org.3

\section{Resumen}

El elote es considerado un alimento tradicional y popular en México; sin embargo, su producción se ha centrado en un número pequeño de diversidad, desplazando principalmente a las variedades criollas. La producción de elote ofrece ventajas económicas respecto a la producción de maíz, la cual, acompañada de innovaciones, mejora la competitividad de los productores en el mercado. El estudio de las innovaciones en la producción de elotes es escaso; por ello, los objetivos de la presente investigación fueron caracterizar la producción de elotes en el estado de Jalisco y determinar el nivel de adopción de innovación y su posicionamiento competitivo. Se encuestó a 14 productores de elote durante los meses de julio a diciembre de 2018 en los municipios de Etzatlán, Ahualulco De Mercado y Guadalajara, Jalisco; cinco proveedores (intermediarios) de elote y cuatro "eloteros" de venta al menudeo. Mediante un análisis jerárquico basado en las variables de adopción de innovaciones, se clasificó a los productores; se determinó el nivel competitivo mediante el análisis de componentes principales (ACP). El índice de adopción de innovación (InAI) calculado como el porcentaje de adopciones que realiza cada productor del total de innovaciones propuestas por categoría, se presentó en un intervalo de 6 al $32 \%$, la categoría con mayor InAI fue sanidad. La mejor posición competitiva se atribuyó a la implementación de innovaciones relacionadas con el manejo de cultivo, mercado y organización; el bajo posicionamiento competitivo se relacionó con un alto índice de innovación de sanidad.

Palabras clave: Alimento tradicional; competitividad; adopción de innovación; producción de elotes

\section{Abstract}

The corn on the cob is considered a traditional and popular food in Mexico; however, its production has focused on a small number of varieties, mainly displacing creole varieties. Corn on the cob production offers economic advantages over corn production, which accompanied by innovations, improves market competitiveness. The study of innovations in corn on the cob production is scarce; therefore, the objective of the present investigation was to characterize the production of corn on the cob in the state of Jalisco, to determine the level of adoption of innovation, as well as to evaluate its competitive positioning. As a case study, in the municipalities of Etzatlán, Ahualulco De Mercado and Guadalajara, Jalisco, 14 corn on the cob producers, five corn on the cob suppliers (intermediaries) and four retail "eloteros" were surveyed. Through a hierarchical analysis, the producers were classified and the competitive level was determined through the analysis of main components (ACP). The innovation adoption index (InAI) of the producers was presented in a range of 6 to $32 \%$, the category with the highest InAI was health. The best competitive position was attributed to the implementations of crop management, market and organizational innovations; a low competitive position was related to a high index of health innovation.

Keywords: Traditional food; competitiveness; adoption of innovation; corn production 


\section{INTRODUCCIÓN}

El maíz (Zea mays L.) es uno de los productos más importantes en los mercados nacionales e internacionales, por sus cualidades alimenticias para la producción de proteína animal, consumo humano y uso industrial (Fideicomisos Instituidos en Relación con la Agricultura-FIRA, 2016). En México, la producción, comercialización y transformación del maíz son una fuente económica importante, debido a que más de cien millones de personas basan su alimentación en este producto, por ello, la relevancia económica y social del maíz en México supera a la de cualquier otro cultivo, y es considerado el cultivo más sensible y, de la agricultura, el que más afecta a la economía campesina (Fernández, Jaramillo, Hernández y Cadena, 2014, Paliwal, Granados, Lafitte y Violic 2001; Hock, Kranz y Renfro, 1989).

El maíz es aprovechado de diversas formas, una de ellas es la producción de elote (estado fenológico lechoso), que representa mayores ventajas respecto al aprovechamiento del grano, debido a que su ciclo de cultivo es más corto y propicia la posibilidad de desarrollar otra siembra si las condiciones ambientales lo permiten; además, una vez cosechado el elote, permite aprovechar el forraje para ensilado o para uso directo en la alimentación del ganado (Fernández et al., 2014). En México, es común el consumo de elote como complemento o ingrediente básico de diversos platillos nacionales (Olivares, 1995).

En los últimos años la problemática en la producción de diferentes productos ha cambiado drásticamente y se refleja en el abordaje actual, no solo atendiendo problemas de producción sino de sustentabilidad, competitividad, diferenciación y recientemente valoración de los recursos asociados a los productos. Por ello, la producción agrícola debe ir paralela a un esfuerzo de innovación sostenido, para mejorar la competitividad en los mercados y favorecer el crecimiento territorial (Pérez, 2019; Ramírez-García, Monterroso-Rivas y Garcia-Espejel, 2019; Méndez, 2006). En México, los estudios sobre innovación en el sector agroalimentario, se han centrado principalmente en la producción primaria (Espejel-García, Illescas-Marín, Hernández-Montes, Santos-Moreno y Ramírez-García 2018a; Espejel-García, Rodríguez, Barrera-Rodríguez y RamírezGarcía, 2018b; Muñoz, Aguilar, Rendón y Altamirano, 2010); particularmente en el maíz las investigaciones y transferencia de tecnología se han centrado en el cultivo y el producto grano. Entre los agentes que intervienen en los procesos de innovación, se resalta la función de los usuarios (Von-Hippel, 1988), las universidades (Mowery \& Sampat, 2005), las multinacionales (Narula y Zanfei, 2005) y recientemente en el ámbito agroalimentario de México destaca el mercado que incentiva y dinamiza los sistemas de innovación (Espejel-García et al., 2014).

Las innovaciones alcanzan su objetivo hasta el momento en que se adopta y se difunde, Pérez y Torreón (2004) definen la difusión como el proceso por el cual una innovación se comunica por varios canales a través del tiempo entre individuos de un sistema y de la innovación que es una idea, práctica u objeto percibido como nuevo por un individuo u otra unidad de adopción. El éxito de las innovaciones se da por la adaptación y empleo de resultados de las actividades de investigación y desarrollo, dentro de un proceso interactivo entre los usuarios, que permita una transferencia 
fácil y eficaz (Ramírez-García et al., 2019; Pérez, 2019; Alburquerque, 2016; Méndez, 2006). En cadenas con alta carga social y enfocadas al autoconsumo, como lo es el maíz en gran parte de México, la proximidad es un elemento estratégico, por lo que una estrategia basada en circuitos cortos representa una alternativa eficiente para comercializar estos productos (Parker, 2005).

El aumento de la población y la urbanización dan como respuesta un incremento en la demanda de productos agrícolas cada vez más diferenciados y dirigidos a estratos de consumidores más exigentes; está creciente demanda de productos agrícolas ofrece la oportunidad para que los agricultores utilicen la producción de elote para mejorar su economía y favorecer la seguridad alimentaria y sobre todo aprovechar las preferencias de consumo de este producto tan emblemático para México. Sin embargo, para satisfacer la creciente demanda, los agricultores necesitan aumentar la productividad de sus cultivos, lo cual implica el uso de insumos tecnológicos, como el implemento de maquinaria especializada, manejo de cultivo y sanidad. Por ello, el objetivo de esta investigación fue caracterizar la producción de elote en el estado de Jalisco y realizar un análisis económico y de innovación, con la finalidad de conocer el posicionamiento competitivo de los productores de elote y factores asociados a su sostenibilidad.

\section{Metodología}

\section{Objeto de estudio}

El estudio fue realizado en el estado de Jalisco, en los municipios de Etzatlán, Ahualulco de Mercado y Guadalajara, donde se recopiló información derivado de la aplicación de encuestas de 14 productores, cinco proveedores (intermediarios) de elote y cuatro eloteros de venta al menudeo; el muestreo utilizado fue no probabilístico de bola de nieve con la finalidad de identificar a los productores y comercializadores potenciales.

\section{Caracterización de la producción de elotes}

Se diseñó una encuesta dirigida a productores de elote la cual se validó previamente con tres productores líderes, con ello se realizaron adecuaciones a la misma; debido a que no se cuenta con un padrón de productores de elote, se entrevistó a los productores con disponibilidad de responder la encuesta y posteriormente ellos referenciaron a otros productores; en la encuesta se les preguntó sobre datos generales, productivos, económicos y de adopción de innovaciones.

El apartado sobre la dinámica de innovación fue integrada por siete categorías: 1) manejo de cultivo, 2) sanidad, 3) nutrición, 4) maquinaria y equipo, 5) tecnologías poscosecha, 6) financiamiento y 7) organización y administración; considerando un total de 28 innovaciones (Manejo de cultivo: Labranza mínima, uso de cultivos de cobertura, asociación de cultivos, uso de semilla mejorada, uso de semilleros nacionales y rotación de cultivos; Sanidad: Control de malezas, control de plagas y control de enfermedades; Nutrición: Uso de abonos agrícolas, análisis de suelos, fertilización balanceada (N, P, 
K), fertilización fraccionada, uso de biofertilizantes, uso de micronutrientes y uso de mejoradores del suelo; Maquinaria y equipo: Camas permanentes, uso de maquinaria especializada, uso de riego tecnificado, nivelación de suelos y uso de sensores infrarrojos; Tecnologías poscosecha: Uso de silos y/o bolsas herméticas; Financiamiento: Ventas por contrato, uso de seguros agrícolas y uso de créditos; Organización y administración: Compras y/o ventas en común, consolidación organizativa y registros técnicos productivos y administrativos. Los datos obtenidos fueron sistematizados en una base de datos empleando el software Microsoft Excel ${ }^{\circledR}$.

\section{Comercialización y manejo del elote}

Se diseñaron dos encuestas, una dirigida a cinco proveedores intermediarios de elote y la otra a cuatro eloteros de venta al menudeo, en ambos casos se les preguntó sobre datos generales, económicos y de ventas. La encuesta a los eloteros de venta al menudeo incluía preguntas respecto al proceso de producción, como criterios de selección, transporte del elote, características del lugar de proceso, acondicionamiento del elote antes de procesarlo, especificaciones del cocimiento del elote y transporte al lugar de venta. Los datos obtenidos fueron sistematizados en una base de datos empleando el software Microsoft Excel ${ }^{\circledR}$.

\section{Análisis de innovación}

El cálculo del Índice de Adopción de Innovación (InAI) se realizó empleando (1) propuesta por Muñoz et al. (2007), donde se asignó a las variables el valor de uno si el productor introdujo la innovación.

$$
\operatorname{InAI}=\sum_{\mathrm{i}=1}^{\mathrm{j}}\left[\frac{\text { Innovaciones }}{\text { Máx.núm.de innovaciones }} \text { readizadas por pos productores }\right] * 100
$$

\section{Análisis económico y posicionamiento competitivo}

El análisis económico de los productores de elote se realizó considerando todos los costos estimados relacionados con la producción del elote (semillas, actividades en realizadas en el cultivo, transporte, certificaciones), además de considerar los ingresos totales brutos obtenidos (toneladas de elote obtenido multiplicada por el precio promedio). Las ganancias económicas se determinaron mediante la metodología propuesta por Espejel-García et al. (2016) empleando (2).

$$
B=\sum_{i=1}^{j} I T-\sum_{i=1}^{j} C T
$$

Dónde: $B=$ Beneficios o ganancias económicas de la producción de elote. $I T=$ Suma de ingresos totales del productor de elote de 1 hasta j. $C T=$ Suma de los costos de producción incurridos en el productor de 1 hasta $j$. 
Posteriormente, se empleó el análisis de clúster con la finalidad de agrupar a los productores en categorías que describen características similares con base al índice de innovación (Rencher, 2002). Se aplicó la técnica multivariada del Análisis de Componentes Principales (ACP) que permite la reducción de variables originales involucradas en el estudio y maximiza su variación generando nuevas variables no correlacionadas entre sí, denominadas componentes principales, se obtuvieron las tendencias en un grupo de datos relacionados con la posición competitiva, para ambas técnicas se empleó el programa XLSTAT Versión 2014.5.03.

\section{Resultados}

\section{Caracterización de la producción de elotes}

La producción de maíz elotero en el estado de Jalisco se caracteriza por tener productores con edades comprendidas entre los 47 y 86 años, con un promedio de 57 años; escolaridad entre primaria y secundaria, con un 43 y $57 \%$, respectivamente. Los productores tienen 16 años de experiencia en la producción de maíz elotero; 36 \% tiene más de 20 años, $43 \%$ de 10 a 20 años y $21 \%$ de ellos tienen menos de 10 años produciendo elote, por lo que se puede decir que cerca $80 \%$ tiene 20 o más años como productor, es decir una generación.

El sistema de producción de elote es en mayor proporción de tipo semi-tecnificado, solo una pequeña parte se produce de manera tradicional, sin incluir ningún tipo de maquinaria. El área sembrada destinada para la producción de elote no es mayor a 5 hectáreas con un rendimiento promedio de 6 ton.ha-1 para el año 2017. De los productores encuestados 64 \% pertenecen a la Comunidad Agraria de Etzatlán o al Ejido de Santa Cruz, lo cual les permite acceder a beneficios como asistencia técnica y créditos.

La producción de elote no es la principal actividad económica de los encuestados, solo es un complemento a su ingreso económico, debido a la temporalidad del cultivo; sus principales actividades económicas son la producción de caña o maíz para grano y la ganadería, sin embargo, la producción de elote proporciona mayor liquidez y un ingreso mayor en comparación con la venta de maíz grano.

\section{Adopción de innovaciones en la producción de elotes}

Los productores de elotes emplean como innovaciones en el manejo del cultivo el uso de especies de cobertera para protección de suelo y fijación de nitrógeno (7 \%), la asociación y rotación con otros cultivos ( 29 y $79 \%$, respectivamente); en el caso de las innovaciones relacionadas con sanidad, un 79 utiliza el control de malezas y $93 \%$ el control de plagas. Las innovaciones de nutrición, como el uso de abonos orgánicos y la fertilización balanceada (N, P, K) son aplicadas por menos de $40 \%$ de los productores; mismo caso se presenta en el uso de equipo y maquinaria especializada. Las innovaciones en la parte de financiamiento incluyen ventas por contrato, uso de seguro y crédito agrícola, las cuales son aplicadas por $21 \%$ de los encuestados. Finalmente, la consolidación organizativa y el registro técnico-productivo y administrativo son aplicados por 14 y $36 \%$ de los productores, respectivamente. 
La eficacia de los sistemas y su continuidad y pertinencia con el mercado puede ser evaluada mediante el nivel de adopción de innovaciones, lo cual conforma una estrategia clave que permite identificar innovaciones con distinto nivel de madurez, funcionalidad y adaptación al sistema (Espejel-García, Ramírez-García y MoraRivera, 2017). En este contexto, el Índice de Adopción de Innovación (InAI) para los productores de elote analizados, tiene un promedio de $18.3 \%$ (Figura 1); el porcentaje de las innovaciones que los productores de elote de Etzatlán y Ahualulco de Mercado aplican, se encuentra por debajo de lo encontrado por Muñoz et al. (2010) para naranja en el estado de Tamaulipas, con un 29 \% de InAI; o lo reportado por Pérez-Velásquez (2017) en naranja en el municipio de Venustiano Carranza, Puebla, con un $21 \%$. Por otra parte, se observa que el InAI no presenta un patrón específico en cuanto a la producción de elotes criollos o híbridos. En la Figura 1 se aprecia el índice de adopción, donde existen tres rangos en el InAI; el primer rango con menos de $10 \%$ en InAI incluye a dos productores, el segundo rango con mayor del $10 \%$ de InAI pero menor a $20 \%$ contiene a seis productores; y finalmente, los productores con más de $20 \%$ en InAI son seis.

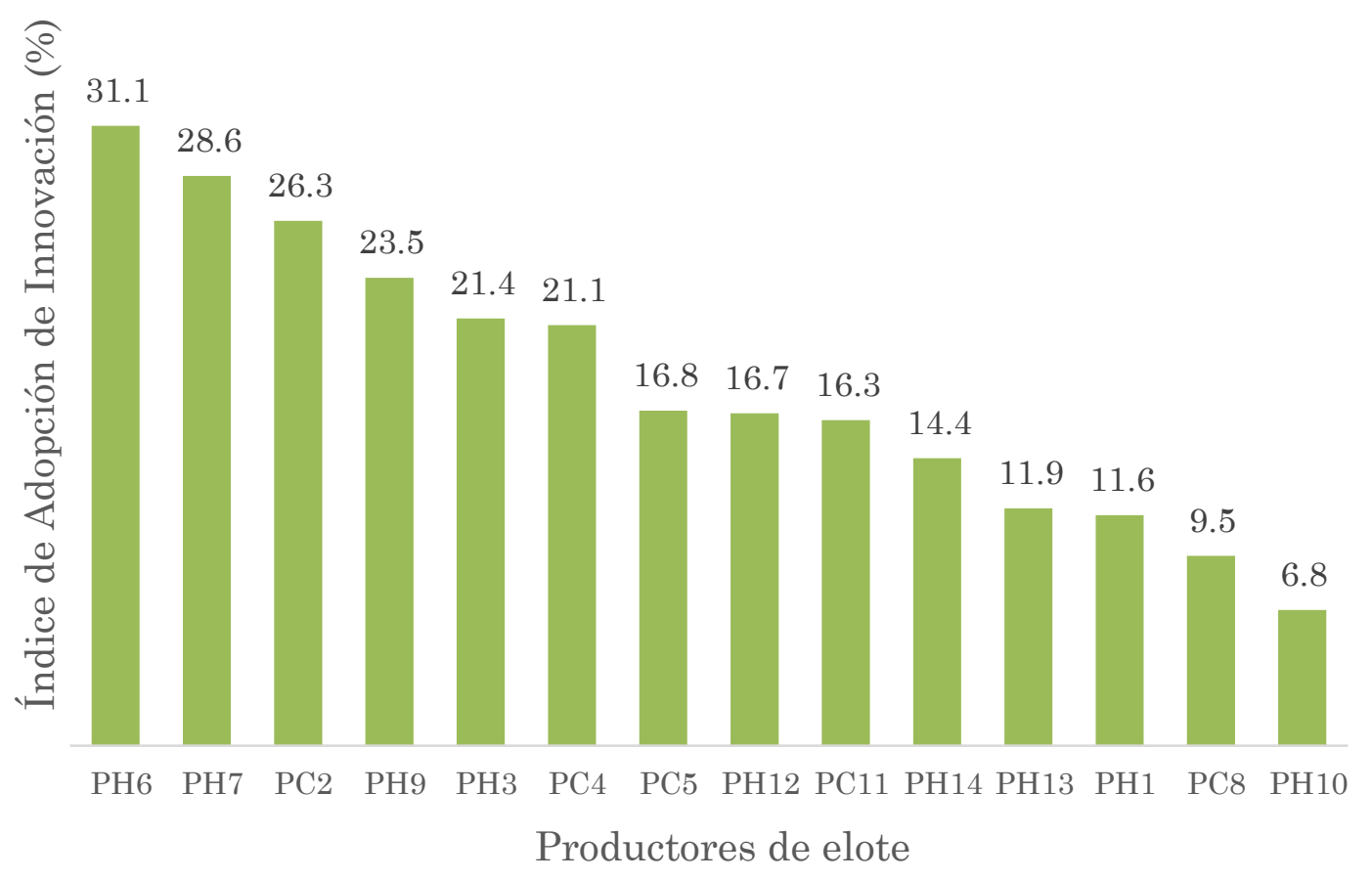

Figura 1. Índice general de adopción de innovación de productores de elote híbrido (PH) y productores de elote criollo (PC) en el estado de Jalisco.

Fuente: Elaboración propia.

En la Figura 2, se aprecia el InAI y la brecha tecnológica promedio para cada una de las categorías evaluadas; la categoría de sanidad es la que presenta el mayor porcentaje de InAI (57\%), esto puede deberse a los problemas ocasionados por plagas, enfermedades y malezas y sugiere una mayor atención en esta área y acudir a diferentes métodos de control, para mejorar los rendimientos y la calidad de los elotes. 


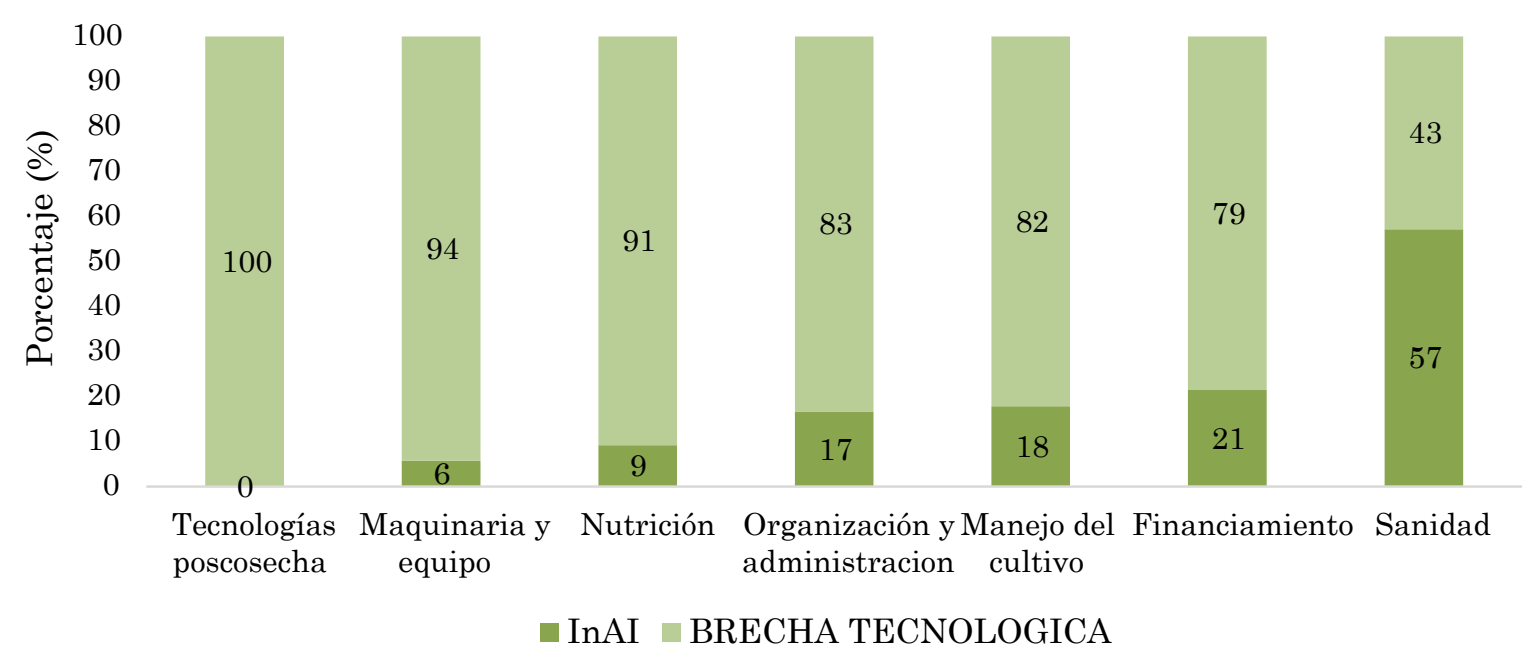

Figura 2. Índice de adopción de innovación y brecha tecnológica promedio para la producción de elotes en el estado de Jalisco.

Fuente: Elaboración propia.

La Figura 3 muestra el promedio del InAI en productores de elote criollo e híbrido; se observa que las categorías de sanidad, financiamiento y manejo de cultivo presentaron índices de adopción de innovaciones más altos en productores de elotes híbridos; las categorías de organización y administración, nutrición y maquinaria y equipo tuvieron mayores índices en los productores de elotes criollos. Diversos investigadores sostienen que el bajo índice de adopción de innovación por parte de los pequeños agricultores se debe a los problemas a nivel de las instituciones y de políticas enfocadas a éste sector (Birner \& Resnick, 2010; Sheahan y Barrett, 2017; Collier \& Dercon, 2014).

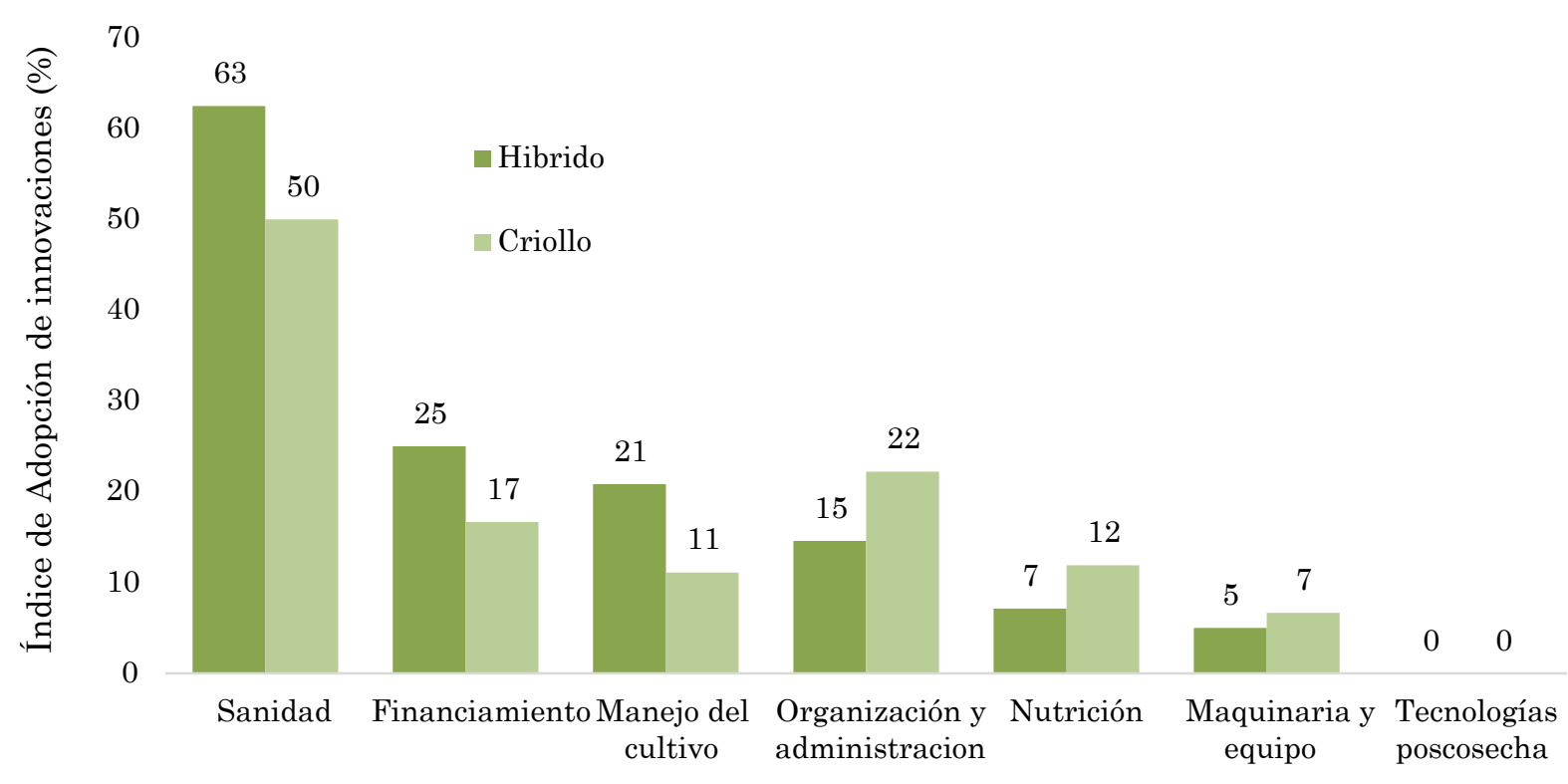

Figura 3. Índice de adopción de innovación promedio para la producción de elotes híbridos y criollos en el estado de Jalisco.

Fuente: Elaboración propia. 
En los procesos de adopción de innovaciones es importante considerar la primera vez que fueron implementadas las innovaciones, así como su evolución. En la Figura 4 se observa la velocidad de adopción de los productores de elote, se aprecia que, del grupo de innovaciones evaluadas, se adoptan por primera vez en el año 2000 tanto para elotes criollos como para híbridos. En el periodo comprendido del año 2000 al 2006, la velocidad del índice de adopción de innovaciones fue superior en productores de elote criollo; sin embargo, a partir del año 2007 la adopción de tecnologías en elote híbrido se incrementó, superando a la implementación de innovaciones para la producción de elote criollo.

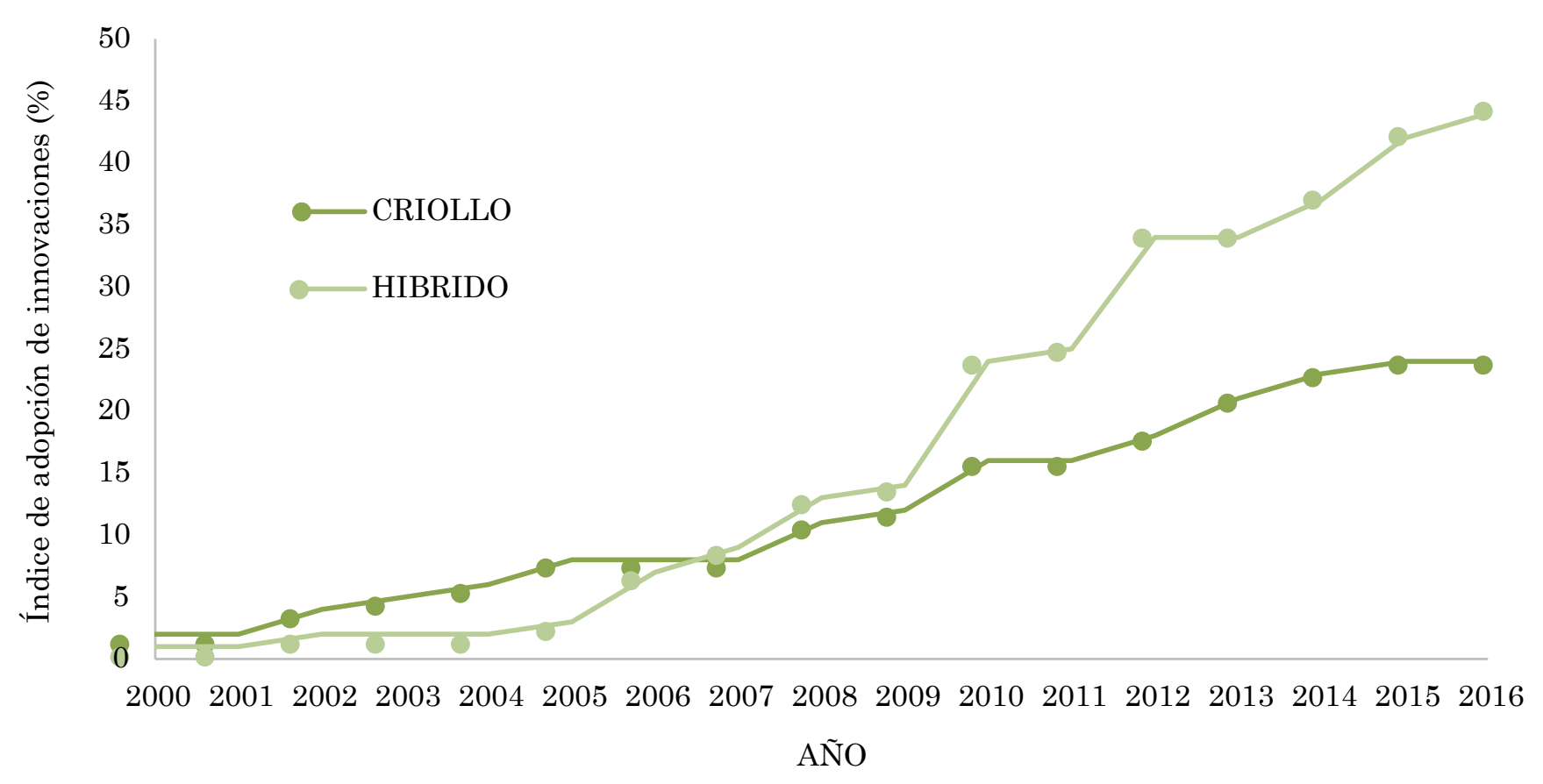

Figura 4. Velocidad del índice de adopción de innovaciones en productores de elote del estado de Jalisco. Fuente: Elaboración propia.

En particular, para los años 2015 y 2016, los productores de elote híbrido implementaron el doble de innovaciones que los productores de elote criollo; lo anterior está asociado con el paquete tecnológico que necesitan los elotes híbridos, en comparación con los criollos.

\section{Posicionamiento competitivo de los productores de elote}

La permanencia de los productores en la producción de elote está relacionada con la capacidad de adaptarse al entorno y con ello lograr una mejor posición frente a sus competidores. Los factores que afectan esta posición de los productores son diversos, por ello, se realizó un análisis de componentes principales considerando algunas variables que desde el punto de vista teórico podrían estar incidiendo, tales como: los índices de adopción de innovaciones y la utilidad de los productores (ingreso y costo total bruto); los resultados indican que con dos factores se explica el $61.4 \%$ de la variabilidad de los datos (indicador Kaiser-Meyer-Olkin = 0.578; Tabla 1). 
TABLA 1.

Estructura factorial del indice de adopción de innovaciones

\begin{tabular}{lrrrrrrr}
\hline & F1 & F2 & F3 & F4 & F5 & F6 & F7 \\
\hline Valor propio & 2.56 & 1.735 & 1.184 & 0.648 & 0.404 & 0.272 & 0.189 \\
Variabilidad (\%) & 36.67 & 24.79 & 16.92 & 9.26 & 5.78 & 3.89 & 2.70 \\
Variabilidad acumulada (\%) & 36.67 & 61.46 & 78.38 & 87.64 & 93.42 & 97.30 & 100.00 \\
KMO=0.578 & & & & & & & \\
\hline
\end{tabular}

Fuente: Elaboración propia.

En la Figura 5 se aprecia la relación entre las categorías evaluadas del InAI y la utilidad; se aprecia que la utilidad esta correlacionada con las innovaciones de manejo del cultivo y financiamiento, las categorías maquinaria y equipo, organización, administración, nutrición se encuentran relacionadas con la utilidad, aunque en menor proporción que las antes mencionadas; el InAI que se encuentra menos relacionado con la utilidad es la sanidad, esto puede deberse a un control de malezas y plagas poco adecuado, lo que ocasiona un mayor costo de producción.

Los Productores de elotes Criollos (PC), presentan un mejor posicionamiento competitivo, debido a las innovaciones de manejo de cultivo, financiamiento, organización, administración y nutrición. Los Productores de elote Híbrido $(\mathrm{PH})$ presentaron mayores índices de adopción de innovación referentes a la sanidad y obtuvieron las menores utilidades. En general, la implementación de innovaciones en manejo del cultivo podría ayudar a los productores de elote, principalmente de elotes criollos, a obtener un mejor posicionamiento frente al mercado.

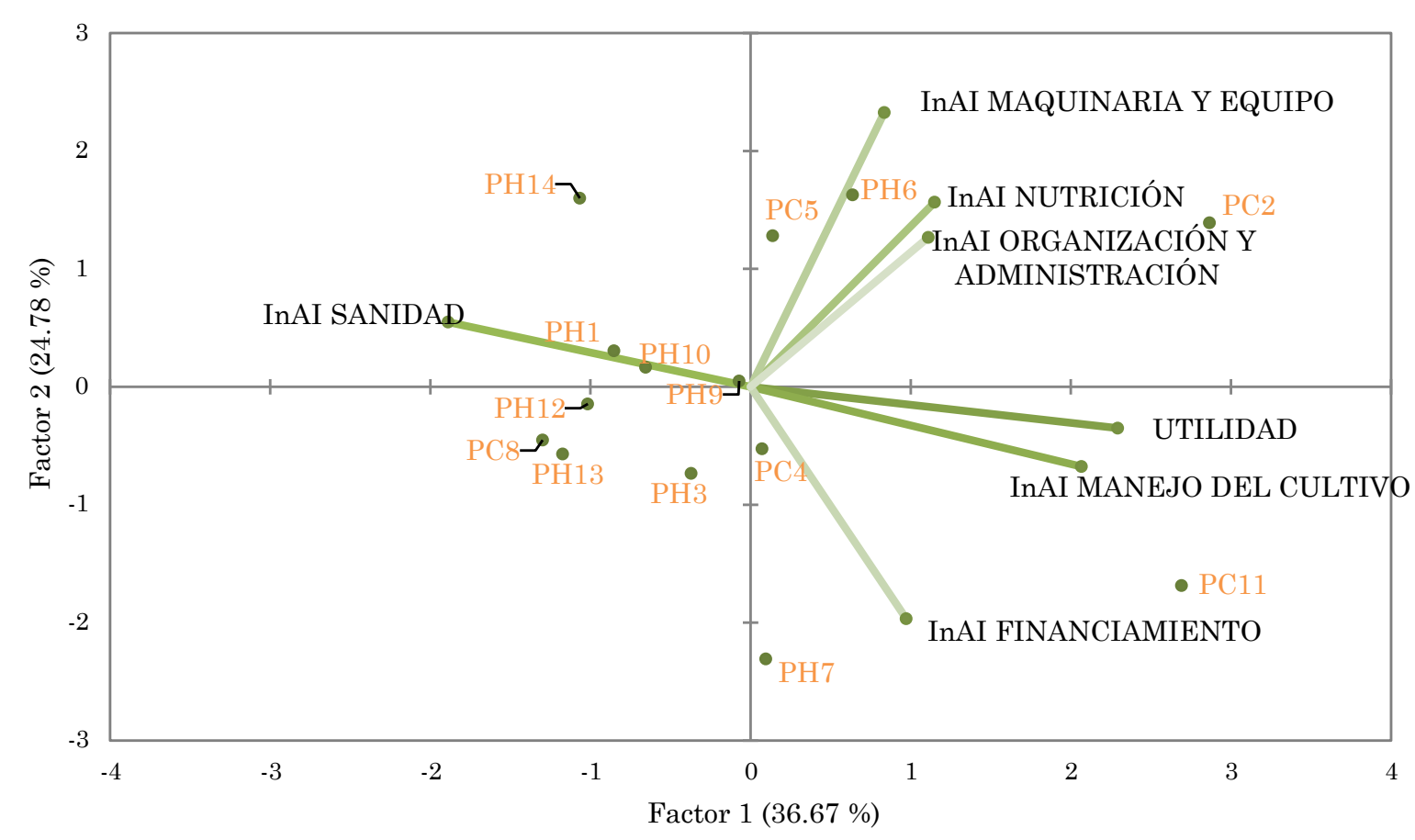

Figura 5. Cargas de los índices de adopción de innovaciones promedio, utilidad y calificaciones de los productores de elote.

Fuente: Elaboración propia. 
Con el análisis de conglomerados jerárquicos se clasificó a los productores en tres grupos (Figura 6) según la variable utilidad e índice de adopción de innovaciones; el primer grupo se encontró conformado por el productor de elote criollo (PC11), quien obtuvo la mejor utilidad y enfoca sus esfuerzos en acceso a financiamiento y manejo del cultivo; el segundo grupo incluyó un productor de elote criollo (PC2); el tercer grupo presentó menor utilidad e InAI, en el cual se encontraron los productores restantes de elote criollo e híbrido, quienes tienen mejores valores en innovaciones relacionadas con la sanidad del cultivo.

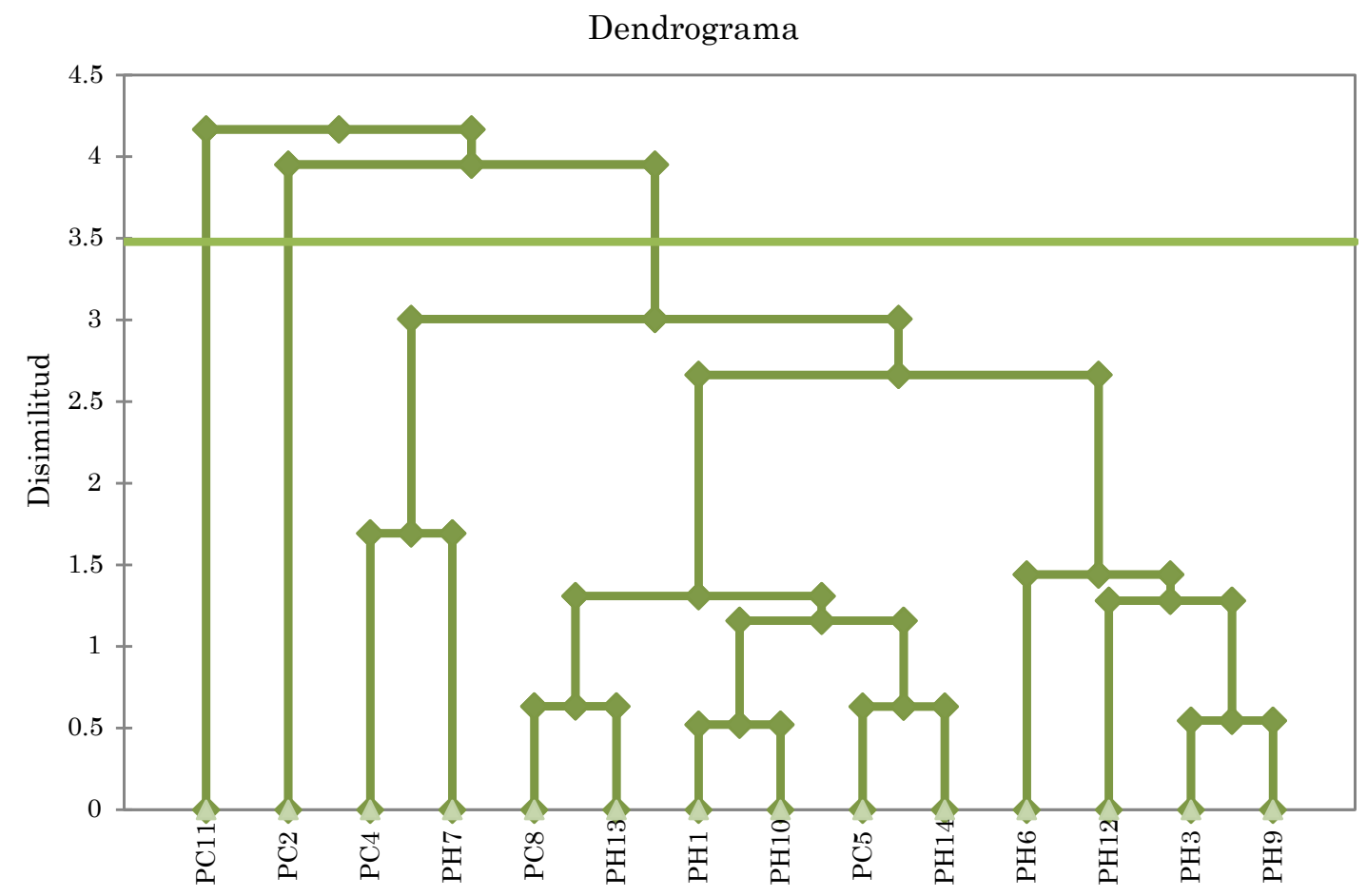

Figura 6. Dendograma de los productores de elote del estado de Jalisco. Fuente: Elaboración propia.

Los productores de elote criollo (PC11 y PC2) son los que obtuvieron mayores utilidades derivados de la producción, esto se debe a que el precio del elote criollo (rojo/ morado) en el mercado en superior al del elote híbrido (blanco), debido a su tamaño y características de sabor que son ampliamente valoradas por los consumidores locales y a la tendencia actual sobre la revalorización de alimentos tradicionales vinculados al origen.

\section{Comercialización de elotes}

La comercialización de los elotes en los municipios de Guadalajara, Etzatlán y Ahualulco es en fresco o previamente cocinados. La comercialización del elote fresco en algunas ocasiones es realizada por los propios productores; sin embargo, existen intermediarios que compran el elote con los productores y lo comercializan en mercados de diferentes municipios, principalmente el mercado del barrio de San José de Analco, Guadalajara. La venta de elotes se realiza durante todo el año, 
proveyendo los elotes de Colima, Jalisco, Michoacán y Sinaloa, principalmente, por ello los intermediarios cuentan por lo menos con cinco proveedores; la temporada con la mayor comercialización de elotes se realiza en los meses de agosto a octubre. Los tipos de elote que se comercializan en la región son blancos, amarillos y rojos. La venta se realiza mayormente al menudeo, por pieza o docena; los elotes comercializados al menudeo se les eliminan las hojas para su venta. El precio de los elotes en el 2018 para elotes rojos y amarillos fue de $\$ 6.00$ por pieza de acuerdo con las respuestas de los comercializadores; los elotes blancos se comercializaron a $\$ 3.00$ la pieza. Los intermediarios contratan empleados asalariados, contrario a los productores que venden directamente su producto, quienes son apoyados por familiares.

Otra forma típica de comercializar el elote en los mercados mexicanos es previamente cocido, las personas que venden el elote de esta forma lo emplean como un ingreso extra a su economía y constituyen negocios familiares pero informales. La antigüedad como comercializadores de elote cocido en las personas entrevistadas no excede los 3 años, quienes cuentan con niveles de escolaridad de secundaria; los elotes con mayor demanda corresponden a los blancos y morados, solo algunos venden elotes amarillos.

La presentación típica de los elotes es hervida o asada y puede ser entero o en grano. El precio del elote cocido oscila entre los $\$ 10.00$ y $\$ 15.00$ pesos mexicanos por pieza de acuerdo con las encuestas; los ingredientes que se le adicionan son: crema, queso, sal, limón y/o chile, lo cual por una parte es más atractivo para el consumidor y por la otra implica un incremento en el precio, incluso dependiendo de la calidad de los ingredientes. La mayoría de los comerciantes solo realiza la venta de elotes cocidos en los meses de julio a octubre, por ser la época de cosecha de elote de temporal.

La calidad del elote es evaluada por indicadores principalmente empíricos y por su tamaño, color de la hoja y su blandura, posteriormente es transportada en bolsas de plástico o canastas al lugar de proceso. En los municipios de estudio, se emplea leña de pino y encino para la cocción, con un periodo aproximado de una hora. La cocción se realiza añadiendo azúcar y sal. La venta puede ser en un solo lugar o pueden realizarse de forma ambulante con carretillas o camiones ofreciendo el elote cocido.

\section{DisCUSIÓN}

Los alimentos y bebidas tradicionales constituyen un componente esencial del patrimonio cultural, la historia, el estilo de vida y la economía local de un país; estos alimentos son aceptados y altamente consumidos por la población local desde hace mucho tiempo, donde los métodos de preparación de dichos alimentos se transmiten de generación en generación (Trichopoulou, Soukara y Vasilopoulou, 2007). El elote es uno de estos alimentos tradicionales en México, su producción en la región estudiada se caracterizó por realizarse en superficies menores de 5 ha, con productores de edades superiores a los 40 años, quienes tienen una producción semi-tecnificada 
y la producción de elotes representa un complemento a la economía familiar. La producción de elotes en la región, principalmente de elotes criollos, se encontró en decremento; estas tendencias concuerdan con las tasas de crecimiento de la producción agropecuaria mundial, las cuales en los últimos años han presentado una disminución, reflejado en la superficie destinada a la producción (Organización de las Naciones Unidas para la Alimentación y la Agricultura-FAO, 2015).

La pérdida observable y continua de la diversidad de los maíces nativos ha surgido el interés por su rescate, conservación y aprovechamiento (Martín-López et al., 2008; Pérez, 2019); el análisis de índice de innovación y competitividad de los productores, puede proporcionar elementos para la revaloración de los elotes, como alimento tradicional; restableciendo con ello, la diversidad en maíces y el aporte económico a familias campesinas, debido a que estas últimas presentan una importante aporte económico por la producción de maíz/elote (Fernández et al., 2014). El bajo porcentaje de aplicación de innovaciones en la producción de elote se debe principalmente a que es un cultivo que contribuye al ingreso económico y la aplicación de otras innovaciones aumentaría el costo de producción, por otra parte, en términos estrictos el sistema de producción de elote funciona de manera sostenible tal como funciona actualmente.

Las innovaciones en productos tradicionales deben realizarse con pertinencia, para aumentar la posibilidad de que cumplan el objetivo y la aceptación por el consumidor sea alta (Guerrero et al., 2009; Guerrero, Claret, Verbeke, Sulmont-Rossé y Hersleth, 2016). En la producción de elotes se observó que la implementación de innovaciones en el área de sanidad fue elevada, sin embargo, ello aumentaba los costos de producción y no presentaba el rendimiento esperado, lo cual ubicaba a los productores con un bajo nivel competitivo. Caso contrario, la utilidad aumentaba cuando el productor aplicaba innovaciones de manejo del cultivo, organización y administración maquinaria y equipo y financiamiento.

Las categorías con los menores InAI están relacionadas con la tecnología poscosecha, maquinaria y equipos y nutrición; la nula adopción en tecnologías poscosecha se debe a la rápida la comercialización del producto y baja perecibilidad, por lo cual, el productor no encuentra en la necesidad de conservar el elote por largos periodos. Las innovaciones aplicadas relacionadas con la nutrición del cultivo se reducen al empleo de fertilizantes que les sobran de otros cultivos o al complemento con abonos orgánicos que aplican con la finalidad de aportar nutrientes al suelo.

\section{Conclusiones}

La producción de elotes en el estado de Jalisco posee características que fortalecen su inserción en los mercados locales, lo cual posiciona al elote como un producto emblemático y típico en su origen, producción y forma de consumirse en México. La adopción de innovaciones está relacionada con el tipo de semilla utilizada lo cual implica un manejo de cultivo diferente y acceso a financiamiento lo que permiten un mejor posicionamiento de los productores. Si bien existen variaciones en cuanto a adopción de tecnologías, los productores analizados obtienen una relación beneficio/ costo mayor a uno, lo que sugiere que es una actividad rentable. 
Los productores de elote criollo son quienes obtuvieron las mayores ganancias de este cultivo debido a que el costo de implementación de innovaciones es bajo y el consumidor valora con un mayor precio a los elotes criollos. Las innovaciones implementadas en el cultivo de elote criollo fortalecen la cadena maíz elote, y permiten vincular a pequeños productores con nichos de mercado que valoran este tipo de productos y pagan más por un producto típico y emblemático que además debe ser criollo.

\section{Reconocimientos y AgRAdecimientos}

Esta investigación fue financiada mediante el proyecto "Transferencia de tecnología e innovaciones en las cadenas de quesos, elotes y mezcal (18026-DTT-62)", de la Dirección General de Investigación y Posgrado de la Universidad Autónoma Chapingo.

\section{REFERENCIAS}

Alburquerque, F. (2016). El enfoque del desarrollo económico territorial y la política. Enseñanzas para el cambio de modelo productivo. En, J. Noguera (Ed.), La visión territorial y sostenible del desarrollo local: una perspectiva multidisciplinar. Territorio, desarrollo, sistema productivo (Vol. 1, pp. 47-66). Valencia: Universitat de València.

Birner, R. \& Resnick, D. (2010). The political economy of policies for smallholder agriculture. World Development, 38(10), 1442-1452. https://doi.org/10.1016/j. worlddev.2010.06.001

Collier, P. \& Dercon, S. (2014). African agriculture in 50 years: smallholders in a rapidly changing world? World Development, 63(C), 92-101. https://doi. org/10.1016/j.worlddev.2013.10.001

Espejel-García, A., Ramírez-García, G. y Mora-Rivera, E. (2017). Empleo e innovación en la producción de queso añejo en el municipio de Zacazonapan. En, Y. V. Chirinos, C. M. Pérez, M. C. Barrios y C. Y. Martínez (Eds.), Universidad, Ciencia, Innovación y Sociedad: desde la perspectiva laboral. Barranquilla-Cúcuta: Editorial Universidad Simón Bolívar.

Espejel-García, A., Barrera-Rodríguez, A. y Cuevas-Reyes, V. (2016). Dinámica de la innovación y ganancias económicas de la producción de leche en el Valle del Mezquital, Hidalgo. Revista Electrónica Nova Scientia, 8(2), 391-408. https:// doi.org/10.21640/ns.v8i17.416

Espejel-García, A., Illescas-Marín, C., Hernández-Montes, A., Santos-Moreno, A. y Ramírez-García, A. (2018a). Innovación y competitividad en la agroindustria artesanal del queso crema de Chiapas. Económicas CUC, 39(2), 25-38. https:// doi.org/10.17981/econcuc.39.2.2018.02

Espejel-García, A., Rodríguez, D. M., Barrera-Rodríguez, A. y Ramírez-García, G. (2018b). Factores estratégicos de la innovación y mercado en queserías artesanales de México. Revista Venezolana de Gerencia, 23(82), 424-441. Disponible en https://produccioncientificaluz.org/index.php/rvg/article/view/23758/24165 
Espejel-García, G. A., Cuevas-Reyes, V., Muñoz, M., Barrera-Rodríguez, A., Cervantes, F. y Sosa, M. (2014). Sistema Regional de Innovación y Desarrollo Rural Territorial; pequeños productores de leche del Valle del Mezquital, Estado de Hidalgo, México. Spanish Journal of Rural Development, 5(2), 1-14.

FAO. (2015). Agricultura mundial: hacia los años 2015/2030. Informe Resumido. [Online]. Disponible en http://www.fao.org/3/y3557s/y3557s00.htm\#TopOfPage

Fernández, I., Jaramillo, J. L., Hernández, J. A. y Cadena, P. (2014). Evaluación agronómica y sensorial de ocho genotipos de maíz (Zea mays L.) para la producción de elote. Agro-Productividad, 7(6), 47-51. Disponible en https://revistaagroproductividad.org/index.php/agroproductividad/article/view/565

FIRA. (2016). Panorama Agroalimentario. Maíz 2016. Dirección de Investigación y Evaluación Económica y Sectorial. [Online]. Recuperado de https://www.gob.mx/ cms/uploads/attachment/file/200637/Panorama_Agroalimentario_Ma_z_2016. pdf

Guerrero, L., Claret, A., Verbeke, W., Sulmont-Rossé, C. y Hersleth, M. (2016). Innovation in Traditional Food Products: Does It Make Sense? In, Ch. Galanakis, Innovation Strategies in the Food Industry (pp. 77-89). Chania: Academic Press. https://doi.org/10.1016/C2015-0-00303-3

Guerrero, L., Guardia, M. D., Xicola, J., Verbeke, W., Vanhonacker, F., Zakowska-Biemans, S., Sajdakowska, M., Sulmont-Rossé, C., Issanchou, S., Contel, M., Scalvedi, M., Granli, B. y Hersleth, M. (2009). Consumer-driven definition of traditional food products and innovation in traditional foods. A qualitative crosscultural study. Appetite, 52(2), 345-354. https://doi.org/10.1016/j.appet.2008.11.008

Hock, J., Kranz, J. y Renfro, B. L. (1989). El “complejo mancha de asfalto” de maíz, su distribución geográfica, requisitos ambientales e importancia económica en México. Revista Mexicana de Fitopatología, 7(2), 129-135. Disponible en https://biblat.unam.mx/es/revista/revista-mexicana-de-fitopatologia/articulo/ el-complejo-mancha-de-asfalto-de-maiz-su-distribucion-geografica-requisitosambientales-e-importancia-economica-en-mexico

Martín-López, J. G., Ron-Parra, J., Sánchez-González, J. J., De la Cruz-Larios, L., Morales-Rivera, M. M., Carrera-Valtierra, J. A., Ortega-Corona, A., VidalMartínez, V. A. y Guerrero-Herrera, M. J. (2008). Caracterización agronómica y morfológica de maíces nativos del noroccidente de México. Revista Fitotecnia Mexicana, 31(4), 331-340. Recuperado de https:/www.revistafitotecniamexicana.org/documentos/31-4/5r.pdf

Méndez, R. (2006). Del Distrito Industrial al Desarrollo Territorial: estrategias de innovación en ciudades intermedias. Desenvolvimento em Questão, 4(7), 9-46. Disponible en https://revistas.unijui.edu.br/index.php/desenvolvimentoemquestao/article/view/120

Mowery, D. C. \& Sampat, B. N. (2005). Universities and national innovation systems. En, J. Fagerberg, D. Mowery and R. Nelson (Eds.), The Oxford Handbook of Innovation, (pp. 209-239). Oxford: OUP. 
Muñoz, R. M., Aguilar, Á. J., Rendón, M. R. y Altamirano, C. J. R. (2010). Análisis de la dinámica de innovación en cadenas agroalimentarias. Chapingo: Universidad Autonoma Chapingo. Disponible en http://repositorio.chapingo.edu. $\mathrm{mx}: 8080 /$ handle/20.500.12098/261

Narula, R. y Zanfei, A. (2005). Globalisation of innovation: The role of multinational enterprises. In, J. Fagerberg, D. Mowery \& R. Nelson (Eds.), The Oxford Handbook of Innovation, (pp. 318-450). Oxford: Oxford University Press. https://doi. org/10.1093/oxfordhb/9780199286805.001.0001

Olivares, M. F. (1995). Estudio de mercado: producción y comercialización del maíz elotero como hortaliza en la región de Tehuacán Puebla [Tesis de Licenciatura]. Universidad Autónoma Chapingo, Chapingo, México.

Paliwal, R. L., Granados, G., Lafitte, H. R. y Violic, A. D. (2001). El maíz en los trópicos: mejoramiento y producción. FAO: Roma.

Parker, G. (2005). Sustainable food? Teikei, Co-operatives and food citizenship in Japan and the UK. [Working Papers in Real Estate \& Planning 11/05]. Reading: University of Reading. Available from http://centaur.reading. ac.uk/21289/

Pérez, C. (2019). Innovación empresarial al servicio de la micro y pequeña empresa nortesantandereana: por la competitividad regional. Economicas CUC, 40(1), 91-104. https://doi.org/10.17981/econcuc.40.1.2019.06

Pérez, M., y Torreón, M. (2004). La teoría de la difusión de la innovación y su aplicación al estudio de la adopción de recursos electrónicos por los investigadores en la universidad de Extremadura. Revista Española de Documentación Científica, 27(3), 308-329. https://doi.org/10.3989/redc.2004.v27.i3.155

Pérez-Velásquez, J. (2017). Análisis de la innovación y comercialización en la cadena agroalimentaria naranja (Citrus sinensis L.) en Puebla [Tesis de Licenciatura]. Chapingo: Universidad Autónoma Chapingo.

Ramírez-García, A., Monterroso-Rivas, A. y Garcia-Espejel, A. (2019). Caracterización de la red de innovación de pequeños productores ganaderos del estado de Sonora, México. Económicas CUC, 40(2), 195-216. https://doi.org/10.17981/ econcuc.40.2.2019.12

Rencher, A. C. (2002). Methods multivariate analysis. New York: John Wiley and Sons, Inc.

Sheahan, M. y Barrett, C. B. (2017). Ten Striking Facts about Agricultural Input Use in Subsaharan Africa. Food Policy, 67, 12-25. https://oi.org/10.1016/j.foodpol.2016.09.010

Trichopoulou, A., Soukara, S. y Vasilopoulou, E. (2007). Traditional foods: a science and society perspective. Trends in Food Science \& Technology, 18(8), 420-437. https://doi.org/10.1016/j.tifs.2007.03.007

Von-Hippel, E. (1988). The Sources of Innovation. New York: Oxford University Press. Available from http://web.mit.edu/evhippel/www-old/books/sources/SofI. pdf 


\section{BIODATA}

Anastacio Espejel García es Doctor en Problemas Económico-AgroindustrialesCIESTAAM; Master en Desarrollo Económico y Cooperación Internacional-Universidad de Murcia España; Maestro en Ciencia y Tecnología Agroalimentaria Universidad Autónoma Chapingo; Ingeniero Agroindustrial Universidad Autónoma Chapingo. http://orcid.org/0000-0002-6462-6681

Carla Zulema Jauregui García es doctorando de la universidad Autónoma de Chapingo, maestra en Ciencias Agroalimentaria por la Universidad Autónoma de Chapingo. Sus intereses de investigación incluyen evaluación sensorial, valoración económica, estudio de los sistemas agroalimentarios y análisis químicos y físico de los alimentos. https://orcid.org/0000-0003-0105-4249

Arturo Hernández Montes es ingeniero agrónomo (1978) de la Escuela Nacional de Agricultura. Maestro en ciencias (1982) de la universidad de Reading. Doctor (1993) de la Universidad de Kansas. Su área de interés para la investigación son alimentos y bebidas tradicionales, estudio de consumidores y aspectos sensoriales de los alimentos. https://orcid.org/0000-0003-1502-3101 